\title{
A Combined Density Functional and Configuration Interaction Method
}

\author{
A. SAVIN \\ Institut für Theoretische Chemie, Universität Stuttgart, Pfaffenwaldring 55, D.7000 Stuttgart 80, FDR
}

\begin{abstract}
Correlation energies are divided into two parts. One contribution is given by a configuration interaction calculation in the space of the natural orbitals with occupation numbers larger than an arbitrary threshold $\nu$. The remaining part is obtained from a $\nu$-dependent functional of the electronic density. Representative examples (for which the existing spin-density functionals fail) are (1) the correlation energies in the He and Be series and (2) the contribution of the correlation energy to the dissociation energy of the first-row dimers. It is shown that even for large values of $\nu$ the errors remain on the order of 0.01 hartree.
\end{abstract}

\section{Introduction}

Since Kohn and Sham [1] showed the possibility of obtaining the correlation energies $E_{\mathrm{c}}$, with density functionals (DF), several applications were carried out for atoms and molecules (see, e.g., Ref. 2). In general, the errors were comparable to those of medium-size configuration interaction (CI) calculations. There are, however, situations where DF show large errors; the most striking failures are perhaps

(1) The incapacity to describe correctly the $Z$ dependence of the correlation energy of the $\mathrm{He}$ and Be series [3]

(2) The inability to reproduce the correlation contributions to the dissociation energies $\left(\Delta D_{e}\right)$ of some first-row dimers (see e.g., Ref. 4).

Another disadvantage of the DF methods when compared with CI appears when an improvement of the calculation is desirable: for DF there is no procedure comparable to enlarging the function space and class of excitations in Cl.

The main cause of the failures of the DF (even with self-interaction or gradient correction) method lies probably in differences between the electron gas and atoms or molecules with respect to orbital level spacings (exclusion and near-degeneracy effects). This has been recognized early by Sinanoglu [5].

The first attempt to include such an effect is due to Tong [6]; he fitted the DF to the homogeneous electron gas with finite electron number.

Lie and Clementi $[7,8]$ found that DF results can be improved considerably by adding the correlation energy obtained in a calculation with a few configurations, introducing the idea that only dynamic correlation is obtained from DF. They did not find, however, a general procedure for the separation of the static and the dynamic part: a double counting of the correlation energy is possible. It has been argued [7] that only special reference functions should be used with DF (see also $[9,10,2]$ ). This 
solution is, however, only provisional, since one soon gets to the point where one would like to add a few more configurations [8].

Another possibility of separating DF from CI contributions is obtained by defining pair correlation energies within DF theory [11]. Such a method assumes, however, that some of the pair energies can be easily obtained within CI calculations.

The first attempt to switch continuously from the usual density functional method $(\mathrm{HF}+\mathrm{DF})$ to the exact calculation by combining $\mathrm{CI}$ with $\mathrm{DF}$ is due to Colle and Salvetti [12]. This was achieved by scaling the electron density with a factor, containing the $\mathrm{HF}$ and the $\mathrm{Cl}$ (or multiconfiguration self-consistent-field, $\mathrm{MCSCF}$ ) two-electron density matrices and an adjustable parameter which ensures a correct treatment of the limiting cases (one determinant and exact wave function, respectively). Despite the arbitrariness of the interpolation procedure, the results obtained by Colle and Salvetti are very encouraging.

More recently, Roos et al. [13] used an effective interelectronic potential (which depends on the averaged density) in connection with MCSCF calculations. Although their correction significantly improves the MCSCF results, they conclude that, for a further improvement, the parameters of their potential should depend on the local density and that this leads to two-electron integrals which must be evaluated numerically.

The present paper may be seen as a continuation of the papers by Lie and Clementi $[7,8]$ : the correlation energy is obtained as a sum of a $\mathrm{Cl}$ and a DF contribution. By the definition of the DF it is possible, however, to avoid a double counting of the correlation energy, without the introduction of any adjustable parameters. The separation of the DF and CI contribution is arbitrary with the exact DF. In practice, however, there are limitations both for obtaining the DF expression and the specific $\mathrm{CI}$ energy. It may be expected that a properly described larger CI space leads to better results as the contribution coming from the approximate DF decreases. Test examples will show that for an accuracy of 0.01 hartree it is sufficient to combine the DF value with the $\mathrm{CI}$ correlation energy contribution coming from near degeneracy.

\section{Method}

Let us start with the definition of the DF for correlation energy as given by Levy [14]. Starting from a HF ground state density $\rho_{\mathrm{HF}}$, he determines the corresponding external potential $v$ by the theorem of Hohenberg and Kohn. Then he constructs the Hamiltonian and calculates the correlation energy. One can go further and obtain also the exact wave function, the exact first-order density matrix, and finally the natural orbitals [15]. A Cl calculation in the space of all natural orbitals with occupation numbers larger than a given threshold $\nu$ yields a contribution to the correlation energy which will be denoted by $E_{>\nu}$; the remaining part of the correlation energy will be $E_{<\nu}=E_{c}-E_{>\nu}$.

Of course, the only use of the prescriptions above is the proof of the existence of the functionals $E_{>_{\nu}}$ and $E_{<\nu}$, which are universal in the sense of Hohenberg and Kohn [15]. In practice, in order to obtain $E_{\mathrm{c}}$, one has to make approximations for both $E_{>v}$ and $E_{<\nu}: E_{>_{\nu}}$ can be obtained from a limited CI calculation and $E_{<\nu}$ from a local (or gradient-corrected) DF. In the limit of large $\nu$ one would get the usual HF + DF case, whereas for $\nu \rightarrow 0$ only a $\mathrm{Cl}$ calculation would be needed. 
The examples presented in this paper use an approximate density functional $E_{<\nu}[\rho]$ obtained from electron-gas calculations. No experimental data were used. The DF used here depends on the threshold $\nu$, but not on spin polarization, as in commonly used DF. The $\nu$ dependence has been introduced only at a level that is approximate even for the homogeneous electron gas (in analogy to the way spin-dependence is usually introduced). The DF describes correctly the homogeneous electron gas when $\nu$ is large. The DF contribution vanishes for small $\nu$. A gradient correction is present. Details on the approximations made are given in the Appendix.

A problem which arises when using such an $E_{<\nu}[\rho]$, is the choice of the threshold $\nu$. Although the distribution of occupation numbers is continuous for the homogeneous electron gas, it is discrete for atoms and molecules. Suppose that the $\mathrm{CI}$ calculation is performed in the space of the first $M$ (approximate) natural orbitals; their occupation numbers are $\nu_{1} \geq \nu_{2} \geq \cdots \geq \nu_{M}$. One could use in principle for $E_{<\nu}$ any value for $\nu$ lying between $\nu_{M}$ and $\nu_{M+1}$. One should better use, however, the latter value. This can be seen from the following example. Let us consider the case of $M$ isolated one-electron systems. In this case $\nu_{1}=\nu_{2}=\cdots=\nu_{M}=1$ and $\nu_{M+1}=$ $\left.\nu_{M+2}=\cdots=0\right)$. The correct result $\left(E_{c}=0\right)$ is obtained only with $\nu=\nu_{M+1}$. With such a choice there is no need for a self-interaction correction of the DF. In the general case, by choosing the threshold equal to $\nu_{M+1}$ some information about the "gap" between the occupation numbers present in molecules is introduced.

The procedure proposed in this paper for obtaining the correlation energy is thus the following:

(1) Do a wave function (CI) calculation in order to obtain

(a) The correlation energy in the space of the first $M$ natural orbitals $\left(E_{>\nu}\right)$

(b) The largest occupation number of a natural orbital not used above, $\nu_{M+1}$

(2) Calculate the value of the density functional $E_{<\nu}$, for $\nu=\nu_{M+1}$ with the total density

(3) Add $E_{>\nu}$ to $E_{<\nu}$ in order to obtain $E_{c}$.

Some details about the calculations follow. The wave function calculations (SCF, MCSCF, or CI) were done with the program systems MELD [17] and MOLPRO [18], installed on the Cray-2 Stuttgart by $\mathrm{H}$. Stoll and U. Wedig.

The Gaussian basis sets are probably better than required for the accuracy needed in the present paper $\left(\approx 10^{-2}\right.$ hartree $)$. They were smaller for the $\Delta D_{\mathrm{e}}$ calculations, larger for the $\mathrm{He}$ and $\mathrm{Be}$ series, and in the atomic calculations where the dependence of the correlation energy on $\nu$ was studied. The exponents used for the symmetries present in HF are those of Ref. 7 (Ref. 19 for He and Ne) with one supplementary diffuse function for each symmetry. The other basis set functions are $(2 p)$ for $\mathrm{H},(4 p)$ for $\mathrm{Li}$ and $\mathrm{Be},(2 d)$ for $\mathrm{B}$ to $\mathrm{F}$ (in the $\Delta D_{\mathrm{e}}$ calculations), $(5 p, 3 d)$ for the He series, $(8 p 4 d)$ for the Be series, $(3 d 2 f)$ for $N$, and $(4 d, 2 f, 1 g)$ for the Ne atom. The contraction coefficients for the $\Delta D_{\mathrm{e}}$ calculations are from Ref. 7 . The basis sets were left uncontracted for the He series. The other atomic calculations were performed with the first four $s$-type functions contracted; for $\mathrm{N}$ and $\mathrm{Ne}$ the first three $p$-type functions were contracted.

The natural orbitals were obtained from a density matrix constructed from a Cr calculation with single and double excitations from a MCSCF calculation by adding to the SCF configuration the near-degenerate ones (when present) in the atomic calculations 
and the configurations given in Ref. 8 (proper dissociation plus the most important unoccupied orbital) for the molecules.

Molecular calculations were performed only at the experimental equilibrium distance [20]. Using other distances (such as HF and MCSCF would only bring changes on the order of $10^{-3}$ hartree.

In order to obtain $E_{>_{\nu}}[\rho]$, the numerical integration was performed in the following way [21]. First the integrand, $f(r)$, is separated into "atomic" contributions by using weight functions, $w_{\mathrm{A}}$ :

$$
\int f(r) d^{3} r=\Sigma_{\mathrm{A}} \int w_{\mathrm{A}} f(r) /\left[\Sigma_{\mathrm{B}} w_{\mathrm{B}}\right] d^{3} r
$$

where $w_{\mathrm{A}}=Z_{\mathrm{A}} \zeta_{\mathrm{A}}^{3} \exp \left(-\zeta_{\mathrm{A}} r\right), Z_{\mathrm{A}}$ is the charge of nucleus $A$ and $\zeta_{\mathrm{A}}$ is a parameter which can be adjusted to give a better fit; our experience [21] has shown that $\zeta_{\mathrm{A}}=Z_{\mathrm{A}}$ is a reasonable choice. Each integral of the sum is then obtained by GaussLaguerre integration over $r$ and Gauss-Legendre integration over $\cos \theta$ and $\phi$.

\section{Results}

The combined density functional plus configuration interaction $(\mathrm{DF}+\mathrm{CI})$ method presented in the preceding section is only useful when small CI (i.e., large threshold values) can be used, as for describing near-degeneracy effects. Furthermore, results should not change drastically when further orbitals are included in the $\mathrm{Cl}$ calculation (i.e., $\nu$ is lowered).

The results of He calculations are shown in Figure 1. The experimental value of $E_{c}$ is 42 mhartree. With the present DF the correlation energy for $\mathrm{HF}+\mathrm{DF}(\nu \rightarrow 2)$ is $71 \mathrm{mH}$. When the absence of natural orbitals down to $\nu=0.0076$ is accounted for,
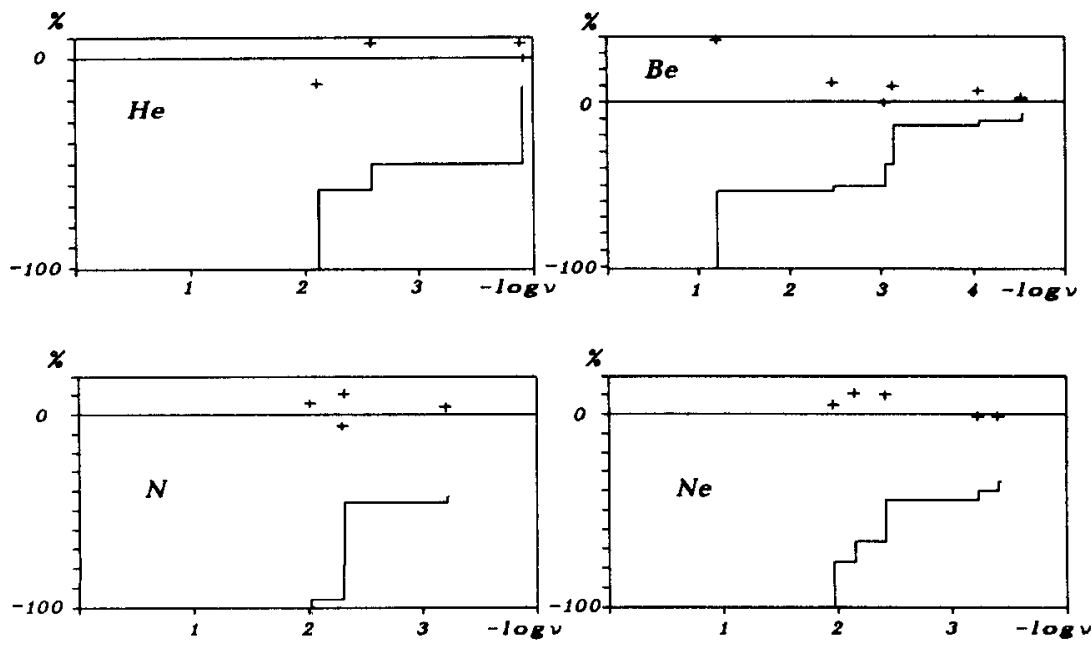

Figure 1. Relative errors in $\mathrm{DF}+\mathrm{CI}$ calculations with different thresholds $\nu$. The staircase function is obtained from a Cl calculation: $100 \cdot\left[\left|E_{>\nu}\right| / \mid E_{c}\right.$ ('experimental') $\left.\mid-1\right]$; the crosses $(+)$ show $100 \cdot\left[\left|E_{>\nu}+E_{<\nu}\right| / \mid E_{c}(\right.$ 'experimental') $\mid-1]$. 
the DF value obtained is $36 \mathrm{mH}$. The reasonable value obtained reflects the absence of near-degeneracy effects. What happens when $\nu$ is lowered? The second natural orbital is also of $s$-type. When included into the $\mathrm{Cl}$ space $16 \mathrm{mH}$ are obtained from the wave function calculation. The DF gives now only $29 \mathrm{mH}$, leading to $45 \mathrm{mH}$ for $E_{c}$. The larger energy contribution comes, however, from the next three (degenerate) natural orbitals, which are of $p$ type. The two contributions are 36 and $9 \mathrm{mH}$, respectively, so that $E_{\mathrm{c}}$ is not changed. Be, N, and Ne results show similar features (see Fig. 1).

In these examples, the $\mathrm{DF}+\mathrm{CI}$ results not only improve the $\mathrm{CI}$ value but are also better than the $\mathrm{CI}$ value obtained by including the next natural orbital in the calculation. Moreover, it is possible that the $\nu \rightarrow 0$ result is worse than that for medium $\nu$, in case the $\mathrm{CI}$ calculation does not properly describe the natural orbitals with small occupation numbers. Of course, there are errors due to the approximation made in the electron gas calculation. Since they are small enough to meet the purpose of this paper, they will not be discussed further.

The correlation energy in the He series is shown in Figure 2. (The DF $+\mathrm{Cl}$ values are obtained by using only the DF value and the threshold given by the second natural orbital.) The experimental value of $E_{c}$ is nearly independent of the nuclear charge, $Z$; DF methods predict a logarithmic increase of $E_{c}$ with $Z$ [3]. For example, the best result for $\mathrm{C}^{4+}$ was obtained up to now with Perdew's gradient corrected DF: $E_{\mathrm{c}}=$ $60 \mathrm{mH}$ instead of $45 \mathrm{mH}$; the error of the same functional is only $2 \mathrm{mH}$ for He. Figure 2 shows that, at least for the ions up to $\mathrm{C}$, this error has been removed by $\mathrm{DF}+\mathrm{CI}$.

An improvement arises also in the Be series (Fig. 2), where the experimental $E_{c}$ increases linearly with $Z$ (related to the near-degeneracy of $2 s$ and $2 p$ [22]). DF predicts a logarithmic increase also in this case [3]. The best result with a DF up to now was again with that of Perdew [23]; the error is, however, over $40 \mathrm{mH}$ for $\mathrm{Ne}^{6+}$ $(0 \mathrm{mH}$ for $\mathrm{Be})$ [23]. The $\mathrm{DF}+\mathrm{Cl}$ values shown in Figure 2 included the $\mathrm{Cl}$ contribution coming from the space spanned by two $s$ and the three degenerate $p$ natural orbitals. They are 0.01 hartree too high both for $\mathrm{Be}$ and for the ions of the series.

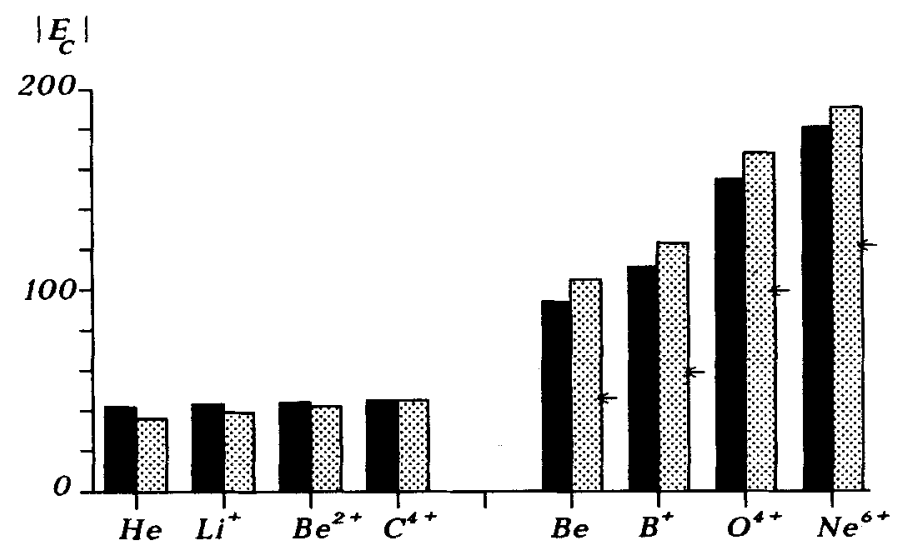

Figure 2. Correlation energies in the $\mathrm{He}$ and in the Be series, in millihartrees. Black bars: experimental; grey bars: DF + CI values; the arrows mark the separation between the DF part

(top) and the cr contribution (bottom). 
Let us now discuss another case in which correlation energy DF render large errors. Although the contributions of the correlation energy to the dissociation energy $\left(\Delta D_{\mathrm{e}}\right)$ are reasonable with DF in some cases, such as $\mathrm{H}_{2}$ or $\mathrm{Li}_{2}$ (errors less than $5 \mathrm{mH}$ with the gradient corrected functional of Perdew [23,4]), others (such as $C_{2}$ ) show errors of 0.1 hartree (with the same functional). It is not even possible to reproduce the trends in $\Delta D_{\mathrm{c}}$ correctly [4] within the first-row homonuclear dimers. Although the experimental values show a maximum for $\mathrm{C}_{2}$, the $\mathrm{DF}$ values have their maximum at $\mathrm{N}_{2}$. (This can be understood from the large density accumulation between the atoms, due to the triple bond.)

What is a good threshold $(\nu)$ for separating the DF and CI parts? In first-row atoms one surely has to include the natural orbitals up to the first $p$ set [22]. The $\nu$ value determined for the atom $\mathrm{X}(\mathrm{X}: \mathrm{Li}-\mathrm{F})$ - it is of the order of $10^{-2}$ - was chosen as an indicator for the dimers $X_{2}$ : the threshold in the molecule $X_{2}$ was chosen as the largest occupation number comparable to the atomic $\nu$. Let us consider $\mathrm{C}_{2}$ as an example. For the $\mathrm{C}$ atom the space to be treated with $\mathrm{CI}$ is given by the first two $s$-type orbitals and by a $p$ set. The occupation number of the next natural orbital is 0.0098 , and $\nu$ was set equal to this number. In the $\mathrm{C}_{2}$ calculation the occupation number of the first $\pi_{\mathrm{g}}$ orbital was 0.0854 , that of the third $\sigma_{\mathrm{u}}$ was 0.0123 , and that of the fourth $\sigma_{\mathrm{g}}$ was 0.0070 . Since the occupation number of the $\sigma_{\mathrm{u}}$ orbital is the largest occupation number comparable to the atomic $\nu, 0.0123$ was chosen as the threshold for $\mathrm{C}_{2}$ calculation. The prescription above is equivalent to limiting the $\mathrm{CI}$ space to the valence one for $\mathrm{N}_{2}, \mathrm{O}_{2}$, and $\mathrm{F}_{2}$; for $\mathrm{B}_{2}$ and $\mathrm{C}_{2}$ the last valence-space $\sigma$-orbital is not used; for $\mathrm{Li}_{2}$ and $\mathrm{Be}_{2}$ some of the " $2 p$ "-orbitals have to be included.

Within this small CI space neither the freezing of the core orbitals nor the improving of the basis set seem to change the results significantly. Although the effect of the former was of the order of $10^{-4}$ hartree $\left(\mathrm{Li}_{2}\right.$ or $\left.\mathrm{F}_{2}\right)$, that of the latter is one order of magnitude larger, as shown by a test calculation in the case of the $\mathrm{N}_{2}$ molecule. After keeping only the first four $s$ and first three $p$ Gaussian contracted and using the $(2 d 1 f)$ functions of Ref. 24 the DF $+\mathrm{Cl}$ result was changed by $1 \mathrm{mH}(8 \mathrm{mH}$ in the MCSCF-CI calculation). Obviously, the space of the natural orbitals with occupation numbers larger than $\nu$ is satisfactorily described with the smaller basis set.

There exist, of course, drawbacks that are due to the limitation of the Cl space. When degeneracy is present, the first-order density matrix is not unique, and thus the natural orbitals have to be defined by a supplementary requirement. One often uses (see, e.g., Ref. 25) the orbitals which are generated by diagonalizing the symmetrized density matrix obtained by averaging the density matrices of the degenerate wave functions [26]. The $\Delta D_{\mathrm{e}}$ values shown in Figure 3 were obtained by assuming axial symmetry not only for the $\mathrm{X}_{2}$ molecules but also for the dissociation products. For isolated atoms one may assume spherical symmetry as well. For $\mathrm{H}, \mathrm{Li}, \mathrm{B}, \mathrm{C}$, or $\mathrm{N}$ this has no effect on the $\mathrm{DF}+\mathrm{Cl}$ correlation energies, since there is no degeneracy present (for $\mathrm{H}, \mathrm{Li}$, and $\mathrm{N}$ ) or due to the fact that the orbital determining $\nu$ is of $s$-type ( $\mathrm{B}$ and $\mathrm{C}$ ). For $\mathrm{O}$ and $\mathrm{F}$ the limitation to the valence space leads to a threshold given by a $p$-type natural orbital. The thresholds are 0.011 and 0.009 (for axial and spherical symmetry, respectively), leading to $260 \mathrm{mH}$ and $253 \mathrm{mH}$ correlation energy ("experimental" $E_{c}: 258 \mathrm{mH}[4]$ ). This difference is larger than the difference that 


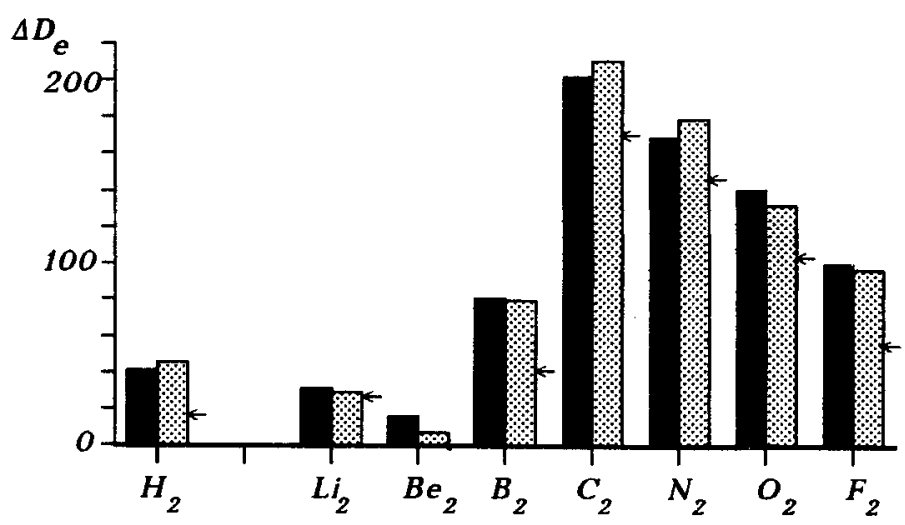

Figure 3. Contributions of the correlation energies to the dissociation energies of the firstrow dimers $\Delta D_{\mathrm{c}}$ in millihartrees. Black bars: experimental; grey bars: $\mathrm{DF}+\mathrm{CI}$ values; the arrows mark the separation between the DF part (top) and the CI contribution (bottom).

arises at the HF level $(2 \mathrm{mH})$. The two values lead, however, to similar errors in $\Delta D_{\mathrm{e}}$ (131 and $147 \mathrm{mH}$, respectively; "experimental" value: $140 \mathrm{mH}$ ). Since the errors in the approximate electron gas calculation used in the present paper are of the same order of magnitude, this problem will not be considered further.

\section{Conclusion}

The aim of the present paper was to show that it is possible to couple a $\mathrm{Cl}$ calculation with an universal spin-independent density functional without doubly counting the correlation energy. In order to test the applicability of such a scheme, an electron gas calculation was used to construct the density functional. Despite the approximations included at this level, a coupling with small-size $\mathrm{CI}$ calculations was shown to lead to reasonable results in cases where existing spin-density functionals fail. In order to study the possibility of reducing the errors in the calculation of the correlation energies beyond the presented level (i.e., a fraction of an electron volt), an improved density functional is needed.

\section{Acknowledgments}

I thank Professors H. Preuss and H. Stoll for continuous support and helpful discussions. I am also grateful to Dr. O. Gunnarsson for useful comments, to Drs. J. Flad and H. Silberbach for reading the manuscript, to B. Miehlich for incorporating the new integration scheme in our program, and to F.X. Fraschio for programming advice. Acknowledgment is also made to the Deutsche Forschungsgemeinschaft for financial support.

\section{Appendix}

The construction of the density functional $E_{<_{\nu}}[\rho]$ will be presented in this appendix. Accurate calculations exist for the correlation energy of the homogeneous electron gas [27], and their accurate parametrization is available [28]. This is used in the local 
density approximation. An improvement of the latter is achieved by using gradient corrections, which are obtained from inhomogeneous electron gas calculations [2931,23 ]. The gradient-corrected density functional is obtained by three-dimensional integration (atomic units are used hereafter):

$$
E_{c}[\rho]=\int\left[\rho \varepsilon_{\mathrm{C}}(\rho)+B(\rho, \nabla \rho)|\nabla \rho|^{2}\right] d^{3} r
$$

$\varepsilon_{\mathrm{C}}$ and $B$ used in this paper are from Refs. 28 and 23, respectively:

$$
\begin{aligned}
& \varepsilon_{\mathrm{C}}(\rho)=A\left\{\ln \left[x^{2} / X(x)\right]+(2 b / Y) \tan ^{-1}[Y /(2 x+b)]\right. \\
& \quad-\left[b y / X(y) \cdot\left\{\ln \left[(x-y)^{2} / X(x)\right]+2[(b+2 y) / Y] \tan ^{-1}[Y /(2 x+b)]\right\}\right. \\
& x=(3 / 4 \pi \rho)^{1 / 6}, \quad X(x)=x^{2}+b x+c, \quad Y=\left(4 c-b^{2}\right)^{1 / 2}, \\
& A=0.0310907, \quad b=3.72744, \quad c=12.9352, \quad \text { and } y=-0.10498 ; \\
& B=\exp [\Phi(\rho, \nabla \rho)] \cdot C(\rho) \cdot \rho^{-4 / 3}, \quad k_{\mathrm{C}}=f \cdot|\nabla \rho| / \rho, \\
& \Phi(\rho, \nabla \rho)=1.745 k_{\mathrm{C}}[C(\infty) / C(\rho)] \rho^{-1 / 6}, \quad c_{5}, \quad c_{3}=2.568 \times 10^{-3}, \quad c_{3}=0.023266 ; \\
& C(\rho)=c_{1}+\left(c_{2}+c_{3} x^{2}+c_{4} x^{4}\right) /\left(1+c_{5} x^{2}+c_{6} x^{4}+c_{7} x^{6}\right), \\
& c_{1}=1.667 \times 10^{-3} ; \quad c_{2}=0.772, \quad \text { and } \quad c_{7}=0.07389 .
\end{aligned}
$$

In Ref. $23 f$ was adjusted to 0.11 in order to obtain - with the DF-the "experimental" correlation energy of $\mathrm{Ne}$. This adjustment is not the best, in the context of the present paper, since one should include some threshold information. Thus, for the sake of simplicity, no fitting to experimental values was performed, and the value chosen for $f$ was the "theoretical" one $[29,30]: f=1 / 6$.

In Eq. (A.1) no dependence on the threshold $\nu$ is present. This has been introduced only in an approximate way (in analogy to the spin-dependence in the local spindensity approximation [28]) namely by using the expression for $\varepsilon_{\mathrm{C}}$ given by GellMann and Brueckner [Eq. (19) in Ref. 32]. In this approximation $\varepsilon_{\mathrm{C}}$ is given by:

$$
\begin{aligned}
\varepsilon_{\mathrm{C}}^{\prime}= & \frac{3}{4 \pi} \alpha^{3} x^{6} \int_{0}^{q_{\max }} q^{3} d q \int_{0}^{\infty} d u\left\{\ln \left[1+Q(q, u) /(\pi q)^{2}\right]-\left[Q(q, u) /(\pi q)^{2}\right]\right\} \\
Q(q, u)= & \int_{s_{\min }}^{s_{\max }} d s \int_{\left(s^{2}+q^{2}-k_{\mathrm{F}}^{2}\right) /(2 s q)}^{1} d \cos \vartheta 2 \pi s^{2}(2 / q) \\
& \cdot[s \cos \vartheta-q / 2] /\left[(s \cos \vartheta-q / 2)^{2}+u^{2}\right] \\
= & \int_{s_{\min }}^{s_{\max }} d s 2 \pi s(1 / q) \ln \left(\left[(s-q / 2)^{2}+u^{2}\right] /\left\{\left[\left(s^{2}-k_{\mathrm{F}}^{2}\right) /(2 q)\right]^{2}+u^{2}\right\}\right)
\end{aligned}
$$

$\alpha=(4 / 9 \pi)^{1 / 3}, q_{\max } \rightarrow \infty ; s_{\min }=\max \left(q-k_{\mathrm{F}}, k_{\mathrm{F}}\right), s_{\max }=q+k_{\mathrm{F}},(s=p+q$ in the notation of Ref. 32).

The correlation energy per particle obtained in the space of all natural orbitals with occupation number larger than $\nu\left(\varepsilon_{>\nu}\right)$ is obtained for the homogeneous electron gas 
by noticing that the natural orbitals are in this case the plane wave states. Thus, the largest momentum that contributes to $\varepsilon_{>\nu}, k_{\nu}$, is known from the momentum distribution, which has been obtained in the present approximation by Daniel and Vosko [33]. It is easily shown that in order to obtain $\varepsilon_{>_{\nu}}$ one only has to replace the integration limits in Eq. (3): $s$ has to be smaller than $k_{v}$. The value of the integral $I=(q / 2 \pi) Q$ for $q<k_{\nu}-k_{\mathrm{F}}$ is

$$
\begin{aligned}
I= & \frac{1}{2}\left(k_{\mathrm{F}}^{2}-q^{2} / 4+u^{2}\right) \ln \left(\left[\left(k_{\mathrm{F}}+q / 2\right)^{2}+u^{2}\right] /\left[\left(k_{\mathrm{F}}-q / 2\right)^{2}+u^{2}\right]\right) \\
& -q u \tan ^{-1}\left[\left(k_{\mathrm{F}}+q / 2\right) / u\right]-q u \tan ^{-1}\left[\left(k_{\mathrm{F}}-q / 2\right) / u\right]+k_{\mathrm{F}} q
\end{aligned}
$$

for $k_{\mathrm{F}}+k_{\nu}>q>k_{\nu}-k_{\mathrm{F}}$ :

$$
\begin{aligned}
I= & \frac{1}{2}\left(k_{v}^{2}-q^{2} / 4+u^{2}\right) \ln \left[\left(k_{v}-q / 2\right)^{2}+u^{2}\right] \\
& -\left[\left(k_{v}^{2}-k_{\mathrm{F}}^{2}\right) / 2\right] \ln \left\{\left[\left(k_{v}^{2}-k_{\mathrm{F}}^{2}\right) / 2 q\right]^{2}+u^{2}\right\} \\
& -\frac{1}{2}\left(k_{\mathrm{F}}^{2}-q^{2} / 4+u^{2}\right) \ln \left[\left(k_{\mathrm{F}}-q / 2\right)^{2}+u^{2}\right] \\
& -q u \tan ^{-1}\left[\left(k_{\mathrm{F}}-q / 2\right) / u\right]+q u \tan ^{-1}\left[\left(k_{v}-q / 2\right) / u\right] \\
& -2 q u \tan ^{-1}\left[\left(k_{v}^{2}-k_{\mathrm{F}}^{2}\right) /(2 q u)+\left(k_{v}-k_{\mathrm{F}}\right)\left(k_{v}+k_{\mathrm{F}}-q\right)\right.
\end{aligned}
$$

whereas for $q>k_{\mathrm{F}}+k_{\nu}, I=0$.

The remaining integrations were performed numerically using QUAD-PACK subroutines [34].

Let us define the factor $\varphi=\left(\varepsilon_{\mathrm{C}}^{\prime}-\varepsilon_{>_{\nu}}\right) / \varepsilon_{\mathrm{C}}^{\prime}$ for further use in obtaining $E_{<_{\nu}}$ : the integrand in Eq. (1) is multiplied by $\varphi$. Thus, the correct behavior is ensured for large and small $\nu$ in the case of the homogeneous electron gas. One should, however, consider the correction for the nonuniform systems, too. This has been done here in a very crude fashion, namely by replacing $k_{\mathrm{C}}$ by $k_{\nu}-k_{\mathrm{F}}$ when the latter is larger than the former. To conclude, the expression for the DF used in this paper is given by

$$
E_{<\nu}=\int \varphi\left[\varepsilon_{\mathrm{C}} \rho+B_{\nu}|\nabla \rho|^{2}\right] d^{3} r
$$

where $B_{\nu}$ is obtained from the expression of $B$, with $k_{\mathrm{C}}$ replaced by $\max \left(f|\nabla \rho| / \rho, k_{\nu}-k_{\mathrm{F}}\right)$.

Presently, the $\varphi$ and $k_{\nu}$ values are obtained by spline interpolation [35]. The density grid is given by 50 points, lying between $x=0.1$ and $x=5.0$. The grid for $\nu$ is given by 20 points, lying between $k_{\nu}=1.01$ and 5 (in $k_{\mathrm{F}}$ units). A plot of $\varphi(\nu)$ for selected density values is shown in Figure 4 (for the dependence of $k_{\nu}$ on $\nu$, see, e.g., Refs. 33 and 36).

The quality of the approximate expression of $E_{<\nu}$ is difficult to assert. The expression for $\varepsilon_{\mathrm{C}}^{\prime}$ gives values that are generally too large by a factor of 1.5 for the interesting range of densities. The values for $k_{v}$ are probably too large, especially for small densities $[37,38]$. The effect on the proportionality factor $\varphi$ remains, however, unclear. Since the DF contributions are normally $\approx 1 \mathrm{eV}$, it may be expected that the errors of the DF are about a fraction of an electron volt. Up to now, the quality of the DF is only tested in atomic and molecular calculations, where there are, of course, also other errors. It seems that the errors of the DF are in the range of $10^{-2}$ for $\nu \approx 0.01$ and an order of magnitude smaller for $\nu \approx 0.001$. The correction of the factor $B$ is 


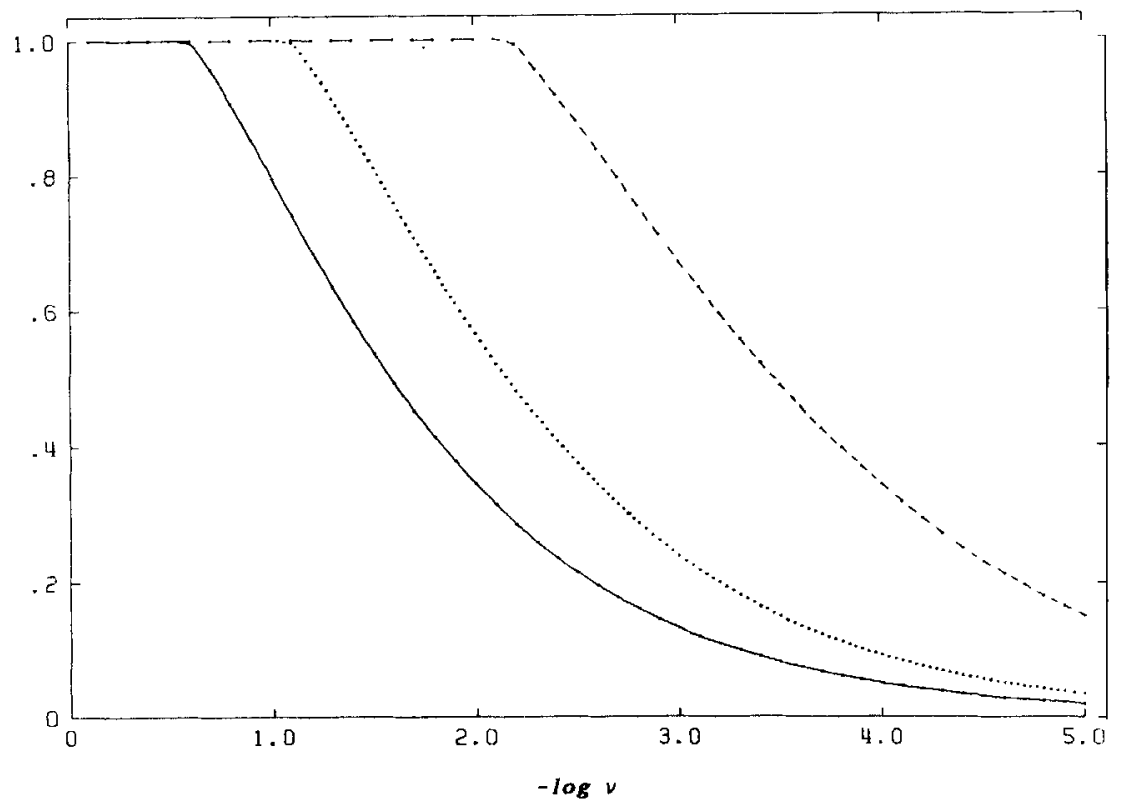

Figure 4. Fraction of the correlation energy not obtainable in the space of the natural orbitals with occupation number larger than $\nu, \varphi$; approximate homogeneous electron gas calculation. Full line: $x=2.2$ ( $\approx$ density at van der Waals surface [39]); dotted line: $x=1.1$ ( $\approx$ maximal valence density of $\mathrm{C}$ atom); dashed line: $x=0.3$ ( $\approx$ density value at Ne nucleus).

less important, especially for energy differences, such as $\Delta D_{e}$; in the case of $\mathrm{O}_{2}$, for example, the change in $\Delta D_{e}$ obtained when substituting $B_{\nu}$ with $B$ is $1 \mathrm{mH}$. Since the DF presently limits the accuracy of the calculation, more accurate calculations for $E_{<\nu}[\rho]$ are desirable.

\section{Bibliography}

[1] W. Kohn and L. J. Sham, Phys. Rev. A140, 1133 (1965).

[2] H. Stoll and A. Savin, in Density Functional Methods in Physics, R. M. Dreizler and J. da Providencia, Eds. (Plenum, New York, 1985), p. 177.

[3] J. P. Perdew, E. R. McMullen, and A. Zunger, Phys. Rev. A23, 2785 (1981).

[4] A. Savin, H. Stoll, and H. Preuss, Theor. Chim. Acta 70, 407 (1986).

[5] O. Sinanoglu, Comm. Atomic Molec. Phys. 1, 116 (1969).

[6] B. Y. Tong, Phys. Rev. A4, 1375 (1971).

[7] G. C. Lie and E. Clementi, J. Chem. Phys. 60, 1275 (1974).

[8] G. C. Lie and E. Clementi, J. Chem. Phys. 60, 1288 (1974).

[9] T. Ziegler, A. Rauk, and E. J. Baerends, Theor. Chim. Acta 43, 261 (1977).

[10] U. von Barth, Phys. Rev. A20, 1693 (1979).

[11] A. Savin, H. Stoll, and H. Preuss, in Local Density Approximations in Quantum Chemistry and Solid State Physics, J. P. Dahl and J. Avery, Eds. (Plenum, New York, 1984), p. 263.

[12] R. Colle and O. Salvetti, Theor. Chim. Acta 53, 55 (1979).

[13] B. O. Roos, M. Szulkin, and M. Jaszunski, Theor. Chim. Acta 71, 375 (1987).

[14] M. Levy, in Density Matrices and Density Functionals, R. Erdahl and V. H. Smith, Jr., Eds. (D. Reidel, Dordrecht, 1987), p. 479. 
[15] P.-O. Löwdin, Phys. Rev. 97, 1474 (1955).

[16] P. Hohenberg and W. Kohn, Phys. Rev. B136, 864 (1964).

[17] L. E. McMurchie, S. T. Elbert, S. R. Langhoff, and E. R. Davidson, NRCC program QC04, obtained by the courtesy of Prof. E. R. Davidson.

[18] W. Meyer, P. Pulay, E. A. Reinsch, and H. J. Werner, MOLPRO program system, obtained by the courtesy of Prof. H. J. Werner.

[19] R. Poirier, R. Kari, and I. G. Csizmadia, Handbook of Gaussian Basis Sets (Elsevier, Amsterdam, 1985), Tables 2.9.1. and 10.32.1.

[20] K. P. Huber and G. Herzberg, Molecular Spectra and Molecular Structure, (Van Nostrand, New York, 1979), Vol. 4.

[21] B. Miehlich et al., to be published.

[22] J. Linderberg and H. Shull, J. Mol. Spectrosc. 5, 1 (1960).

[23] J. P. Perdew, Phys. Rev. B33, 8822 (1986).

[24] R. Ahlrichs, P. Scharf, and K. Jankowski, Chem. Phys. 98, 381 (1985).

[25] E. R. Davidson, Rev. Mod. Phys. 44, 451 (1972).

[26] W. Kutzelnigg, Z. Natf. 18a, 1058 (1963).

[27] D. M. Ceperley and B. J. Alder, Phys. Rev. Letters 45, 566 (1980).

[28] S. H. Vosko, L. Wilk, and M. Nusair, Can. J. Phys. 58, 1200 (1980).

[29] D. C. Langreth and D. J. Mehl, Phys. Rev. Lett. 47, 446 (1981).

[30] D. C. Langreth and D. J. Mehl, Phys. Rev. B28, 1809 (1983); Phys. Rev. B29, 2310 (1984).

[31] C. D. Hu and D. C. Langreth, Phys. Scripta 32, 391 (1985).

[32] M. Gell-Mann and K. A. Brueckner, Phys. Rev. 106, 364 (1957).

[33] E. Daniel and S. H. Vosko, Phys. Rev. 120, 2041 (1960).

[34] R. Piessens, E. de Doncker-Kapenga, C. W. Überhuber, and D. K. Kahaner, QUADPACK-A Soubroutine Package for Automatic Integration (Springer-Verlag, Berlin, 1983).

[35] G. Engeln-Müllges and F. Reutter, Formelsammlung zur numerischen Mathematik mit StandardFORTRAN 77-Programmen (Bibliographisches Institut, Mannheim, 1986).

[36] J. Lam, Phys. Rev. B3, 3243 (1971).

[37] L. J. Lantto, Phys. Rev. B22, 1380 (1980).

[38] E. Pajanne and J. Arponen, J. Phys. C: Solid State Phys. 15, 2683 (1982).

[39] R. F. W. Bader, W. H. Henneker, and P. E. Cade, J. Chem. Phys. 46, 3341 (1969).

Received May 6, 1988 